\title{
A Process Model for Establishing Business Process Crowdsourcing
}

\author{
Nguyen Hoang Thuan \\ Can Tho University of Technology \\ Vietnam \\ nhthuan@ctuet.edu.vn
}

\section{Pedro Antunes}

Victoria University of Wellington

\section{David Johnstone}

Victoria University of Wellington

\section{Abstract}

Crowdsourcing can be an organisational strategy to distribute work to Internet users and harness innovation, information, capacities, and variety of business endeavours. As crowdsourcing is different from other business strategies, organisations are often unsure as to how to best structure different crowdsourcing activities and integrate them with other organisational business processes. To manage this problem, we design a process model guiding how to establish business process crowdsourcing. The model consists of seven components covering the main activities of crowdsourcing processes, which are drawn from a knowledge base incorporating diverse knowledge sources in the domain. The built model is evaluated using case studies, suggesting the adequateness and utility of the model.

Keywords: Business Process Crowdsourcing, Crowdsourcing, Design Science, Process Model

\section{Introduction}

Organisations have been opening their boundaries to access external labour, knowledge, expertise, and innovation for some time, including the use of open software, open innovation and outsourcing. More recently, crowdsourcing, referring to the distribution of work to Internet users via open calls, has emerged as a viable alternative (Howe 2006). By adopting crowdsourcing, organisations can, for the first time, procure, assign and control work online independently from geography, time, and location. Crowdsourcing can also tap into external expertise and creativity. Such capability, combined with an elastic and round the clock workforce, increases organisational agility and resilience. All in all, the virtually limitless workforce makes crowdsourcing an attractive strategy to organisations (Gill et al. 2015; Rosen 2011; Saxton et al. 2013).

While bringing new business opportunities to organisations, crowdsourcing at the same time changes the way organisations conceive and manage work. Through crowdsourcing, the organisational boundaries become hard to identify since organisations may access an unprecedented range of human resources from virtually everywhere (Tranquillini et al. 2015; Vukovic and Bartolini 2010). Furthermore, crowdsourcing has changed the way organisations design work structures. Different from traditional structures, which establish a stable and topdown hierarchy of responsibilities and remunerations, crowdsourcing promotes a dynamic, loose and bottom-up structure where professional knowledge, decision making, problem solving, and supervisory control can be assigned to unknown members of the crowd (Brabham 2013; Kittur et al. 2013). These changes properly reflect a new, perhaps more turbulent, organisational business model fostered by the Internet era.

In this new business model, one important challenge is what the best way to establish a crowdsourcing process is. We stress here the etymology of the word 'process', which refers to a systematic and repeatable course of action to accomplish some deliberate results. Wellplanned and dedicated processes are assumed not only to produce better crowdsourcing 
results, but also to deploy the strategy faster on top of existing crowdsourcing platforms (Tranquillini et al. 2015). In contrast, an ad hoc process affects organisations due to the need for re-planning and the waste of organisational resources (Muhdi et al. 2011; Rouse 2010). Motivated by the challenge, coupled with the increasing popularity of crowdsourcing as a business practice, this paper aims at supporting organisations successfully establishing the crowdsourcing process.

Given the important role of the process view in crowdsourcing strategies, researchers have already begun to investigate the crowdsourcing process. Although different studies have already investigated several parts of the crowdsourcing process, most of them tend to focus on its individual aspects (Geiger and Schader 2014; Man-Ching et al. 2011). We note in particular that a large number of studies regard crowdsourcing as a one-off venture, instead of being one among many business processes available to an organisation. This one-off view leads to scattered, ad hoc practices, which are often difficult to reproduce, support and optimise.

Recently, some research efforts have focussed on integrating and harmonising concepts of crowdsourcing processes (Amrollahi 2015; Hetmank 2013), yet further efforts are needed to validate and empirically test these propositions before they can be used in practice. For this reason, there is a current lack of a holistic crowdsourcing process model, which organisations can rely upon to establish crowdsourcing processes. Against this gap, the key research question addressed in this paper is stated as follows:

What is the best way to model the holistic crowdsourcing process, which identifies, structures and relates its fundamental components in a systematic way?

This research will investigate crowdsourcing using a Business Process Management (BPM) lens, essentially decomposing complex systems into a set of independent, yet coordinated, activities (van der Aalst and Hee 2004). This lens allows us to analyse independently crowdsourcing components and coordinate them into an integrated crowdsourcing process. We designate this particular view as Business Process Crowdsourcing (BPC), which was first coined by La Vecchia and Cisternino (2010). Based on the BPC view, we then seek to identify the salient activities of crowdsourcing processes and use them to build a process model that supports the establishment of crowdsourcing as an organisational business process. Finally, we evaluate the adequateness and utility of the proposed model by examining two case study projects.

Balancing between building and evaluating, we adopt design science as the research paradigm (Hevner and Chatterjee 2010; Hevner et al. 2004). Design science comprises two important complementary activities: build and evaluate (Hevner and Chatterjee 2010). The build activity focuses on developing artefacts to address the defined problem and to meet the identified business needs. The evaluation activity then assesses the artefacts to form evidence for the artefacts' utility (Venable et al. 2012). Following the tenets of design science, we first build a knowledge base using a scoping literature review. The model is designed using the raw materials provided by the scoping review, offering a systematic comprehensive approach to model construction. The evaluation is done using two case studies of crowdsourcing projects, where the proposed model becomes an analytical lens investigating the collected data drawn from multiple sources that include interviews with key members of the projects. As a result, the case studies provide empirical evidence about utility and adequateness of the proposed model.

The expected contributions of the current study are threefold. First, the study provides holistic understanding of the crowdsourcing process with the BPC concept (Geiger et al. 2011; ManChing et al. 2011). Second, we propose a model for BPC that identifies the critical components that organisations should consider when integrating a crowdsourcing strategy. By adopting a process-centred perspective, the model addresses the current lack of a way to organise business processes based on crowdsourcing (Khazankin et al. 2012a; Satzger et al. 2011). Third, through the case study approach, the study evaluates the adequateness and utility of the model in two crowdsourcing projects. The case studies provide empirical evidence that complements other 
research efforts seeking to conceptualise the crowdsourcing process (Amrollahi 2015; Hetmank 2013; Thuan et al. 2014).

The remainder of this paper is structured as follows. The next section discusses the related work, providing an overview on crowdsourcing and defining the concept of BPC. We then present a detailed development of the proposed model, starting with a scoping review to identify the main activities of crowdsourcing processes. Subsequently, we describe the two case studies and present results from using the model as an analytic lens. We finally discuss our findings and conclude the study, including suggestion for future research directions.

\section{Background}

\subsection{Concept of Crowdsourcing}

The concept of crowdsourcing has recently emerged when Howe (2006) introduced a process utilising the crowd for fulfilling tasks through the Internet. Investigating this concept, researchers proposed several underpinnings behind the emergence of crowdsourcing. By and large, these underpinnings can be grouped into three categories: the wisdom of crowd, the dominance of Web technology, and the organisational context.

First, it is now widely accepted that the crowd's wisdom plays a significant role in crowdsourcing (Saxton et al. 2013; Zhao and Zhu 2014). The 'wisdom of crowds' was explored by Surowiecki (2004), who claimed that "under the right circumstances, groups are remarkably intelligent, and are often smarter than the smartest people in them" (p. xiii). Malone et al. (2010) have extended the underpinning by adding the idea of collective intelligence. Different from the 'wisdom of crowds' that is based on the independence of individuals (Surowiecki 2004), the idea of collective intelligence stresses the coordination of many individuals (Bonabeau 2009; Malone et al. 2010). This extension allows crowdsourcing to find solutions in not only individual capability but also coordinated ways.

The second category of underpinnings concerns Web-centric platforms, such as social media and community sites, which have changed the roles of Internet users from passive receivers to more active contributors (Brabham 2010). Brabham (2013) notes that Internet users seem keen to contribute their ideas, knowledge, skills, and labour into these platforms. Such contributions are as well valuable for crowdsourcing (Saxton et al. 2013). From an organisational perspective, the Web empowers the open call, which allows reaching any given interested participants, which is a distinctive characteristic of crowdsourcing (Doan et al. 2011; Schenk and Guittard 2011). With these characteristics, the Web provides a medium for organisations to approach a large number of users who actively contribute to different work propositions.

The last underpinning comes from an organisational standpoint. We have already mentioned that Web-centric platforms enable the crowd to contribute with fewer barriers, e.g. regarding time and space. Therefore, the next question is whether organisations need such contributions. In fact, they do. The needs for external agents to solve organisational problems have been clearly presented in both outsourcing (Dibbern et al. 2004) and crowdsourcing literature (Muntés-Mulero et al. 2013). By adopting crowdsourcing, organisations may get benefits similar to outsourcing, such as cost savings and access to outside capabilities (Rouse 2010; Saxton et al. 2013), or even more, such as customer involvement and flexible, on-demand labour.

Given the aforementioned discussion, a combination of the crowd, Web technology, combined with organisational demands, can explain the emergence of crowdsourcing. However, it seems the demands of organisations for crowdsourcing have recently changed. Previously, organisations used crowdsourcing mainly for simple one-off tasks (Zhao and Zhu 2014). Recently, they have started to adopt crowdsourcing for more complex organisational processes, such as product development and industrial processes (Djelassi and Decoopman 2013; Muntés-Mulero et al. 2013). This leads to the need for clear conceptualisation of business process crowdsourcing. 


\subsection{Crowdsourcing Process and Business Process Crowdsourcing}

A crowdsourcing process structures a set of activities necessary to operationalise a crowdsourcing project. Understanding the crowdsourcing process allows us to understand its activities, and thus possibly produce better crowdsourcing results (Tranquillini et al. 2015). Consequently, this process view has attracted some attention from researchers. By and large, research on crowdsourcing processes can be classified into two views: high and low level of granularity. With high granularity, a few studies adopt a holistic conceptualisation about the crowdsourcing phenomenon. Trying to paint an overall picture of the crowdsourcing process (Muhdi et al. 2011; Stol and Fitzgerald 2014), they tend to focus more on high level concepts, and thus face significant gaps explaining what is the best way to effectively establish the crowdsourcing process.

In the other view, a larger number of studies tend to investigate only specific parts of a crowdsourcing process. The ad hoc nature of these studies has been recently highlighted in the literature (Geiger and Schader 2014; Man-Ching et al. 2011; Zhao and Zhu 2014). The issue is not about their usefulness due to lack of repeatability, but instead there is no collective cohesiveness. As a result, there is little scaffolding of the studies' outcomes towards a structured, holistic framework. That is, this group of studies have suggested scattered sets of practices, which challenges organisations when trying to establish their crowdsourcing processes. As a result, the domain is still lacking "a comprehensive guideline through which practitioners can initiate and manage their crowdsourcing projects" (Amrollahi 2015, p. 2).

In our research, we attempt to reconcile the two views by providing a more integrated picture of the crowdsourcing process. More precisely, we describe this integration as Business Process Crowdsourcing (BPC). The term BPC was coined by La Vecchia and Cisternino (2010), and further discussed by Thuan at al. (2014) as a way to establish organisational business processes based on crowdsourcing. Etymologically, BPC combines the phrase business process with the word crowdsourcing. This paper elevates the business process construct to be equally important to the crowdsourcing construct. According to Aalst and Hee (2004), a business process is defined as a collection of individual activities and a workflow coordinating them. It aims to achieve a particular goal with both effectiveness and efficiency. A business process is purely conceptual, yet it serves as a template for creating multiple, real life instances of the same process, which organisations may create repeatedly and concurrently. Given that, we define BPC as a set of activities completed by crowdsourcing entities, in conjunction with a coordination of these activities, that collectively form the entire business process.

Our thesis is that BPC proposes an integrated approach for organisations to establish crowdsourcing processes both systematically and efficiently. More precisely, the BPC perspective allows analysing both individual aspects of crowdsourcing and coordinating them into an organisational workflow (La Vecchia and Cisternino 2010; Lüttgens et al. 2014). Furthermore, BPC also allows to standardise crowdsourcing processes. Process standardisation can only be achieved by comprehending all related activities and their relationships. BPC that relies on both the individual and coordinated views provides a unique position for this comprehension. In that sense, BPC is expected to establish crowdsourcing as a repeatable organisational practice, and move crowdsourcing process to a more well-defined status.

Given this central role, there are many calls for further studying BPC, especially how to conceptualise it. Vukovic et al. (2010) was among the earliest researchers that asked "how does crowdsourcing become an extension of the existing business process" (p. 7). Khazankin et al. (2012a) echo the question and complained about "the lack of an integrated way to execute business processes based on a crowdsourcing [platform]" (p. 1). Similarly, Lüttgens et al. (2014) have recently emphasised the need to build a dedicated process for crowdsourcing. The need of BPC can also be implied from the fact that organisations have utilised crowdsourcing for some of their core, complex processes (Djelassi and Decoopman 2013; Muntés-Mulero et al. 2013), which should be necessarily linked with other internal business processes. 
Addressing these calls, we now move onto the BPC focus with a process model. Only a few models/frameworks of crowdsourcing processes have been proposed in the field, but they focus more on technical aspects of crowdsourcing systems rather than the business processes operated on these systems (Hetmank 2013). Furthermore, most of the models proposed so far have not yet been empirically evaluated (Amrollahi 2015). Taking that into consideration, this study aims to propose and evaluate a process model establishing BPC.

\section{A BPC Process Model}

The current study follows the design science paradigm to build and evaluate a process model for BPC (Hevner and Chatterjee 2010; Hevner et al. 2004). Laying out the foundations for this type of research, Hevner et al. (2004) suggest that research should be based on a sound knowledge base, which can be drawn from three sources: scientific theories, existing artefacts, and experiences and expertise. Yet, crowdsourcing is an emerging field lacking a strong theoretical background (Zhao and Zhu 2014), which makes it difficult, if not impossible at this stage, to rely on the first source. Given that, the study must rely on the other sources of knowledge. Adopting a design science method grounding existing artefacts and experience and expertise (Thuan et al. 2015), the study begins with an inventory of existing artefacts and experience and expertise in the crowdsourcing field. For this purpose, we adopt a scoping method to extract domain knowledge from the existing crowdsourcing literature. We then synthesise the process model from the extracted domain knowledge.

\subsection{Scoping Knowledge Sources}

Adopting a scoping review that enables a comprehensive view on the literature of a certain topic (Paré et al. 2015), we analysed the crowdsourcing literature to identify the main components of the crowdsourcing process. The detailed steps are described in our conference paper, and interested readers are directed to (Thuan et al. 2014). We summarise, here, its main outcomes. The scoping review started by systematically searching sources related to crowdsourcing from popular online bibliographic databases. We filtered out the irrelevant sources and sharpened the pool into 536 sources. The next step involved content analysis to identify relevant components of the crowdsourcing process, and to eliminate sources not contributing with at least one component. As a result, we ended up working with 238 reviewed sources.

The reviewed sources were then analysed to identify the main components of crowdsourcing processes. In particular, we employed an analytical process based on coding (Miles et al. 2014). We started with an abstract level of analysis, centred on three high-level activities: decision to crowdsource, design, and configuration. We then extracted, coded and analysed the main components and detailed features of crowdsourcing processes. Coding was done iteratively, which required extracting, comparing, and merging data multiple times. The detailed coding process can be found in (Thuan et al. 2014).

\subsection{Main Stages and Activities of Crowdsourcing Processes}

The end result of the analysis revealed a diversity of sub-activities and associated features, which were then aggregated into a set of logical components. We found more than 20 components, and identified the number of sources supporting each component. The "wisdom of researchers' suggests taking into consideration the number of supporting sources to indicate the important components. When applying the 'wisdom of researchers', we chose a cut-off value where components with less number of supporting sources are filtered out. This choice faced a trade-off. If the cut-off value was low, many components would be selected and the model would become too complex, which is undesirable for a conceptual model (Jonker and Pennink 2010). In contrast, if the value was high, only a few components would be selected, which reduces the representation level of the model. Testing several values, we finally chose 10 as the cut-off value that balances the aforementioned trade-off. Consequently, we eliminated from the list components with less than 10 supporting sources. The final list is shown in Table 1. Details of these components are clarified in the next sections. 


\begin{tabular}{lc}
\hline Components & $\begin{array}{c}\text { No. of supporting } \\
\text { Sources (n>10) }\end{array}$ \\
\hline Quality control & 42 \\
Incentive mechanism & 37 \\
Crowd management & 32 \\
Task design & 29 \\
Results aggregation & 26 \\
Workflow design & 25 \\
Capability \& characteristics of crowdsourcing & 23 \\
Task assignment & 21 \\
Output & 17 \\
Platform & 16 \\
Technical configuration & 16 \\
Circumstance to crowdsource \& decision factors & 16 \\
\hline
\end{tabular}

Table 1: Main components of business process crowdsourcing

\subsection{BPC Model Construction}

Further analysis of the components identified in the previous sections lead us to construct the process model. We followed guidance from Webster and Watson (2002) for building conceptual models from extant literature. Once again, the three high-level activities, i.e. the decision to crowdsource, design, and configuration, were used. More precisely, the components listed in Table 1 were each allocated to one of the high-level activities. The allocations on the decision to crowdsource and configuration were straightforward, because they exhibit strong conceptual links. For instance, components 'circumstance to crowdsource and decision factors' and 'characteristics of crowdsourcing' are logically linked to the decision to crowdsource (Thuan et al. 2016). 'Technical configuration' is also clearly linked to the configuration activity.

However, allocations to the design activity were more difficult since the links extracted from the reviewed sources are more diffuse and cover very different concerns. To help logically organise these components, we classified them into 'what' and 'how' categories, where the former focuses on identifying what work has to be done, and the latter focuses on how work is expected to be done. The 'task design' and 'workflow design' components are related to the 'what' category, while the remaining components, including 'crowd management', 'quality control' and 'incentive mechanism' relate to the 'how' category. In particular, crowd management includes profiling the members of the crowd, which, for instance, includes how to approach and manage the expected crowd; quality control includes mechanisms to control the quality; and the incentive mechanism concerns a decision on how to reward the crowd workers. Using the classification described above, we allocated the 'what' components before the 'how' components, drawing from the conceptualisation of the outsourcing process described by Dibber et al. (2004). These components were further expanded to comprise the activities that organisations go through as they progress through their crowdsourcing processes. This is depicted in Figure 1 as the BPC process model. 


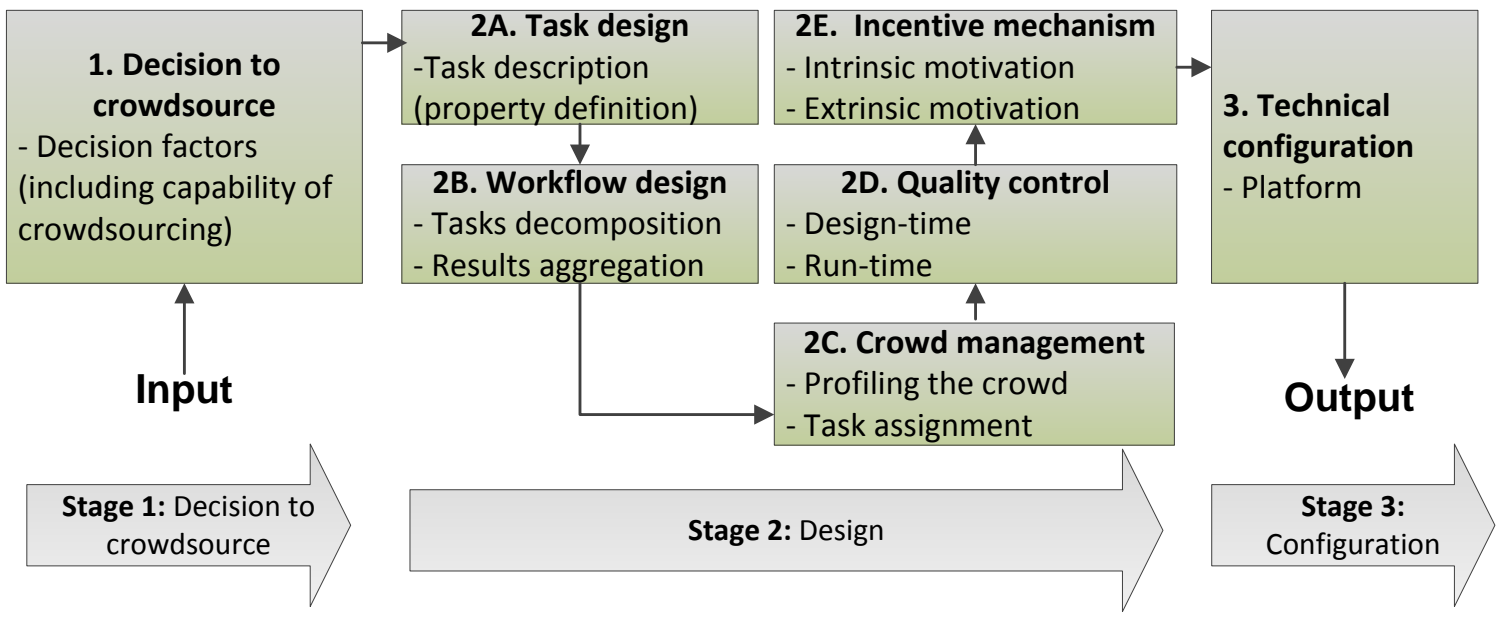

Figure 1: Process model of business process crowdsourcing

\subsection{BPC Model Description}

We now describe the process model in more detail. As seen in Figure 1, the model adopts the input-process-output (Pedersen et al. 2013) and stage-gate configurations (Cooper 2008) that are typical of process models. More precisely, the model consists of seven components structured into three stages, which are described as follows.

Decision to crowdsource. The crowdsourcing process is triggered by an opportunity to crowdsource a piece of work, which starts with a decision to crowdsource (Muhdi et al. 2011; Wexler 2011). This component initially conceptualises the crowdsourcing strategy in order to "decide whether the crowdsourcing approach is appropriate to solve their internal problem/problems [tasks]" (Muhdi et al. 2011, p. 322). The component is a logical antecedent to any crowdsourcing project, similar to the 'make or buy' decision in outsourcing projects (Dibbern et al. 2004). By making it explicit in the model, we signal that the decision to crowdsource should be founded on a logical assessment of the project adequacy.

To make a logical decision to crowdsource, organisations need to evaluate several contingency factors. Thuan et al. (2016) have identified several factors influencing the decision to crowdsource. Analysing 50 papers related to the decision, these authors suggest nine influencing factors, which have been structured into a comprehensive decisional framework considering the task, people, management, and environment. That study also derives a set of actionable guidelines for logically making the decision, which are completely applicable to our model. Due to the limited space, we do not describe them here and point readers to the abovementioned reference.

Design. After the decision to crowdsource has been made, the stage 2 covers a set of design activities necessary to operationalise the decision. It includes five components: task design, workflow design, crowd management, quality control, and incentive mechanism. Task design component transforms the conceptual ideas about the crowdsourcing tasks into concrete task descriptions (model component 2A). Most of the reviewed sources suggest clearly defining what tasks are crowdsourced (Malone et al. 2010; Rosen 2011). The aim of this component is to designate a complete task description that can be given to the potential crowd members who may perform the tasks. To define these tasks, the task properties like significance, autonomy, variety, etc. suggested by Tokarchuk et al. (2012) should be taken into account.

The next component concerns the workflow design. This involves task decomposition and results aggregation (model component $2 \mathrm{~B}$ ). Task decomposition divides the tasks into a set of smaller tasks. This activity has been suggested by several researchers to increase the potential number of workers interested in participating in the open call (Afuah and Tucci 2012; Kulkarni et al. 2012). A counterpart to task decomposition is results aggregation. Results aggregation describes how the outputs from the smaller tasks will be put together so that the objectives of 
the overall task may be fulfilled (Geiger et al. 2011). The results aggregation largely depends on task decomposition, as the function of the former is to reverse the results of the latter. Kittur et al. (2013) explained this relationship that "facilitate[s] decomposing tasks into subtasks, managing the dependencies between subtasks, and assembling the results" (p. 5).

Crowd management is a design component that refers to how organisations manage the crowd members in order to accomplish the defined tasks (model component $2 \mathrm{C}$ ). The reviewed sources suggest two sub-components of crowd management: profiling the crowd (Allahbakhsh et al. 2012) and assigning tasks (Khazankin et al. 2012b). First, organisations analyse the required capacity of crowd members for performing a task (Allahbakhsh et al. 2012; Kittur et al. 2013), and use this evaluation to build member profiles. Based on these profiles, organisations can determine an overall picture of the crowd and may impose constraints to crowd recruitment (Chandler and Kapelner 2013; Stewart et al. 2010). Second, based on the crowd profiles, task assignment can be executed. That is, tasks can be assigned to crowd members who have appropriate profiles. Examples of existing task assignment mechanisms include the auction-based mechanism (Satzger et al. 2011) and the scheduled mechanism (Khazankin et al. 2012b).

According to Table 1, quality control is the most frequently cited and thus should be regarded as a critical component (model component 2D). One distinctive characteristic of crowdsourcing is that tasks are performed by crowd members with very different backgrounds, skills and expertise (Hirth et al. 2012). This sometimes leads to a number of low-quality contributions. Thus quality control mechanisms are necessary to ensure the outputs meet the organisation's quality goals (Allahbakhsh et al. 2013; Ipeirotis et al. 2010). By and large, quality control mechanisms can be classified into design-time and run-time (Allahbakhsh et al. 2013). At design-time, organisations can design tasks and workflows in a robust way to increase the chances of receiving high-quality contributions (Eickhoff and De Vries 2013). At run-time, organisations can consider several active quality control mechanisms like expert reviews, peer reviews, gold standards, output agreements, and even majority voting (Allahbakhsh et al. 2013).

Crowdsourcing relies on voluntary members of the crowd to perform tasks. Thus, organisations need incentive mechanisms to attract and engage these voluntary members in their open calls (model component 2E). The reviewed sources suggest that incentive mechanisms should be developed based on two main types of motivation: intrinsic and extrinsic. For extrinsic motivation that focuses on workers' external drives, most of the investigated sources have examined the adoption of financial incentives (Kaufmann et al. 2011; Mason and Watts 2009). Regarding intrinsic motivation that focuses on workers' internal drives, a variety of factors have been suggested by the extant literature, such as fun (Doan et al. 2011), meaningful tasks (Chandler and Kapelner 2013), and love of the community (Kaufmann et al. 2011).

Configuration. The final stage focuses on how to configure a crowdsourcing process for instantiation in computational systems. Since this activity mainly concerns an in-depth technical view (e.g. adopting specific architectures, technical frameworks, and computational platforms), the business perspective adopted by this study limits our considerations regarding this component to considering available crowdsourcing platforms. Furthermore, we note that several tools supporting the technical aspects of the configuration process have already been proposed, including Turkit (Little et al. 2010), Crowdforge (Kittur et al. 2011), and BPMN4Crowd (Tranquillini et al. 2015). We expect that, in the near future, tools may be able to automatically transform a designed crowdsourcing process into an instantiation capable of running on specific crowdsourcing platforms. As a result, we regard the main output of this component as a configuration file containing a set of low-level details about the crowdsourcing process, which should be supported by existing crowdsourcing tools and platforms. 


\section{BPC Model Evaluation with Case Studies}

After the construction of the process model as a design science artefact, we now have to evaluate the proposed model. We decided to evaluate the model using case studies for three reasons. First, case studies enable artefacts to be evaluated in their practical environments. Second, case studies can be used to explore the complex nature of crowdsourcing by providing in-depth, detailed explanations about their components and overall structure. Yin (2013b) states that "for evaluations, the ability to address the complexity and contextual conditions nevertheless establishes case study methods as a viable alternative among the other methodological choices" (p. 322). Third, case studies are considered appropriate for evaluating design science artefacts, as suggested by Gill et al. (2015) regarding the evaluation of a social architecture framework.

\subsection{Approach}

For the case study evaluation, we considered the model regarding to two metrics: adequateness and utility. Adequateness is defined as 'the degree to which the components and their arrangement in the model align with the activities done in the studied crowdsourcing project'. We define utility as the 'usefulness of the model as perceived by the study participants'. Using these two metrics, we collected, analysed and compared data from two crowdsourcing projects. We followed the guidelines provided by Yin (2013a; 2013b) for conducting case study evaluation research, including how to select cases, collect data, and analyse data.

\subsection{Case Selection}

The selection of crowdsourcing projects was based on comparability and access to source material. First, we selected projects with a comparable team size, between 2 and 10 members. This range is sufficiently large to include multiple project roles, which the model aims to support, but not so large as to hold a diversity of settings that overshadow the evaluation purposes. Second, we chose crowdsourcing projects where we had access to project participants and other data sources. As a result, two crowdsourcing projects were selected: Crowd Tagging (CT) and Logo Design Contest (LDC).

The CT project was part of a bigger project aiming to uncover the impact of New Zealand predators on biodiversity in urban areas. This CT plan involved the installation of motiontriggered cameras in 40 locations in New Zealand, which collected more than 65,000 pictures. CT aimed at identifying the animals captured in these pictures. Because of the large number of pictures that needed to be analysed, the project launched a website with an open call to help tagging the pictures. Project development involved a team of three members: project manager, web developer, and consultant. The call was live from June to December 2014. As a result, the project attracted over 300 users. About half of them tagged more than 20 pictures each.

The other project LDC utilised the crowd for artistic design. A University in the Mekong delta, Vietnam was founded in 2013 from what began as a tertiary education centre. As a result of this transformation process, the University needed a new logo that would represent the spirit of the University. To design the logo, the University adopted a crowdsourcing approach that opened the logo design to designers from both inside and outside the University. It was in this spirit that the LDC project was created. The project started in May 2013 and finished in December 2013, when the winning logo was officially adopted by the University. When the project was launched, it received 68 logo designs from the crowd. Three of them were selected and declared as the winning solutions: two were awarded for creative prizes and one was awarded for the final winning solution, which is the current logo of the University.

\subsection{Data Collection}

We collected data from multiple sources, both primary and secondary. Secondary sources included press releases, the open calls, meeting reports, and project websites, all of which provided materials necessary to clarify key project activities. The activities and their relationships were further detailed and validated in interviews. Across the two case studies, we conducted three key informant interviews with project leaders and other participants, both 
face-to-face and through Skype. Due to the small size of the project teams, these interviewees had to 'wear many hats' and therefore could provide insights into several perspectives of the crowdsourcing projects. Besides being interviewed about the activities performed in the projects, the interviewees were asked to analyse a printed version of the model proposed in this paper and were asked to make a judgment and produce comments about the usefulness of the model. A summary of demographic information about the cases is presented in Table 2.

\begin{tabular}{lll}
\hline & Crowd Tagging & Logo Design Contest \\
\hline Number of project members & 3 & 10 \\
Project duration & 6 months & 7 months \\
Project purpose & $\begin{array}{l}\text { To tag pictures about } \\
\text { animals in New Zealand }\end{array}$ & $\begin{array}{l}\text { To design a logo for the } \\
\text { University }\end{array}$ \\
Interviews & 1 & 2 \\
Roles of interviewees & - Project leader & - Project leader \\
& & - Project coordinator \\
Other data sources & - Press and media & - Press and media \\
& - Website, tutorial & - Internal documents (e.g. \\
& - Internal documents \\
& example submissions) & meeting reports, example \\
& submissions) \\
\hline
\end{tabular}

Table 2: Demographic information

\subsection{Empirical Analysis}

To prepare data for analysis, we first arranged a full description of each case, including details about the project, project team, and project activities. We then used the model proposed in this paper to map the project activities into the model components, while critically analysing the interviewees' comments about the model. More precisely, this empirical analysis included the two following activities.

Adequateness analysis: This analysis followed a pattern matching technique (Yin 2013a), which looked for major similarities, patterns, and notable differences between the model and the activities reported for each project. To begin, we examined each case description for phases indicating the activities of crowdsourcing processes. We marked phases or sentences describing the project activities, and matched them to the model components. Success to match suggested a similarity, while failure to match suggested a potential difference. To maintain the independence, matching was undertaken by the first author and checked by the other authors. This process generated a list of tentative matching patterns. The matching patterns were then cross-checked using the interviews and supplementary materials, which allowed us to refine the existing patterns and to identify additional patterns that emerged from the interviews. As a result, the final list of matching patterns (both similarities and differences) was created, allowing us to finally map the project activities in the model for comparing between them (presented in Figures 2 and 3 below).

Utility analysis: We gathered judgements and comments from the interviewees regarding the perceived utility of the model. During the interviews, we asked "what do you think about the model components?' The interviews were analysed using template analysis, a simple and well-known procedure (King 2012). The analysis started with a few predefined codes related to utility, including 'usefulness', 'future use' and 'future improvement', which were then applied to the interview transcripts. We reviewed the transcripts and identified transcript snippets relevant to our codes. During this process, some emerging codes were created to further capture the judgements made by the interviewees. The codes were then aggregated and synthesised into salient templates, which will be discussed in the results section. 


\subsection{Validity}

Validity is critical factor in qualitative evaluation since it allows judging levels of accuracy and strengthening knowledge claims that the research represents. Yin (2013a) shares similar views on determining validity in case studies when considering construct validity, internal validity, external validity, and reliability. He further suggests several useful techniques for establishing validity. The current study was already applied the techniques, summarised in Table 3.

\section{Construct validity}

- Use multiple sources of evidence

- Case study reports reviewed by key project members

\section{Internal validity}

- Insights from the crowdsourcing literature

- Pattern matching in data analysis

\section{External validity}

- Use literal replications to choose the cases

\section{Reliability}

- Use case study protocol

- Develop and maintain case a study database

Table 3: Study validity

\subsection{Case Study Results}

The case study results are structured according to the two investigated metrics, adequateness and perceived usefulness.

\subsubsection{Adequateness of the Model}

To report on model adequateness, we graphically represent the project activities of the two cases using the model as a baseline. This highlights not only the similarities but also the differences between our model and the investigated projects. Figures 2 and 3 summarise the projects' activities. Differences with the model are represented in italic; Sub-activities are presented in smaller font size and marked with '+'.

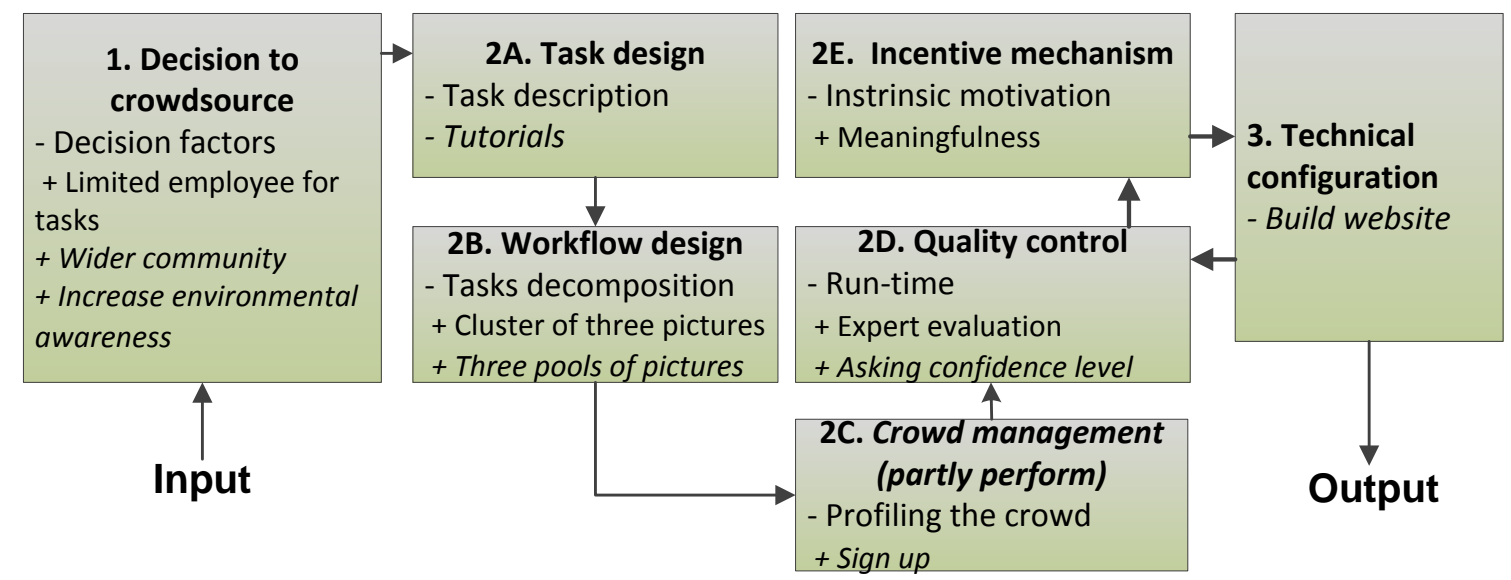

Figure 2: Activities of Crowd Tagging (CT) 


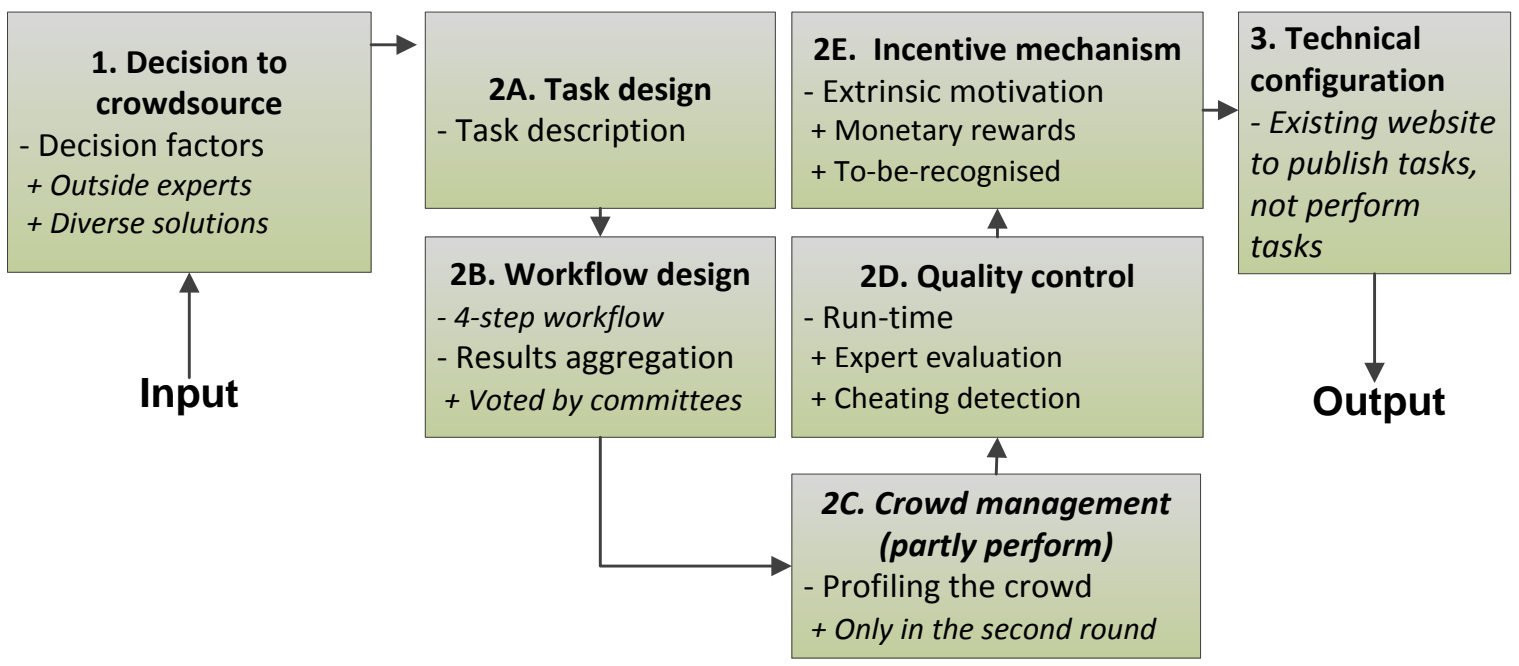

Figure 3: Activities of Logo Design Contest (LDC)

Based on this graphical representation, we observe high adequateness of the model components. Both representations show strong concordance between the model components and the projects' activities. Examples include the strong alignment on decision to crowdsource, task design, workflow design, incentive mechanism, quality control, and partial alignment on crowd management and technical configuration. Several project sub-activities are also aligned with the model. However, both cases reveal several additional activities that were necessary to instantiate the components in practice. Examples include developing a tutorial in the task design of the CT case, and aggregating results through voting in the workflow design of the LDC case. Nevertheless, we find a strong alignment between the model components and the two projects, which suggests high adequateness.

Regarding specifically the interdependencies suggested by the model, the two investigated projects are also largely aligned, i.e. they generally adopt the sequence of steps from input, decision to crowdsource, several aspects of crowdsourcing design, configuration, and finally to output. This alignment is stronger in the LDC case where most components follow the model sequence. In the CT case, we find strong alignment in the first four components, but some differences in the relationships among the last three components. More precisely, the last three were developed in a more iterative way, rather than following a sequential relationship. More details about the activities' interdependencies are presented below.

\section{Crowd Tagging (CT)}

The CT project started with an input consisting of a large number of pictures to be analysed. To process these pictures, the project manager decided to adopt crowdsourcing. He stated three supporting reasons: 1) limited human resources to process the vast amount of data; 2) allowing wider community to access the collected data; and 3) increasing environmental awareness of the community. The first reason, which was considered the most important decision factor by the project manager, examined the lack of internal employees to perform the task (Afuah and Tucci 2012; Malone et al. 2010; Thuan et al. 2016). Reason 1 is consistent with the 'decision to crowdsource' model component, while reasons 2 and 3 were specific to the nature of $\mathrm{CT}$ as a citizen science project.

After deciding to crowdsource, the project manager specified the crowdsourcing process itself, starting with task design. A task description was developed to promote the general aims of the project and explaining how the task could be fulfilled by the crowd: "this research aims to evaluate the use of remote cameras to estimate abundances of non-native predators in urban environments. You will be shown a series of images, taken earlier this year, from various cameras placed around the Wellington city and asked to identify the animal in the photograph" [CT, Website]. The task design is consistent with the model component 2A. Besides, we note the project included a tutorial and a visual explanation of the task, which served to train the 
crowd on how to perform the tagging. Such focus on training seems appropriate for this type of task, and research suggests training the crowd may improve results (Park et al. 2014).

The CT project designed the crowdsourcing workflow through task decomposition. First, the whole activity was divided into sub-tasks of tagging three pictures, which the project alluded to as a cluster. This clustering was directly related to how data was collected in the project: "the camera takes three pictures every time they detect something. Thus, the group of three pictures helps make the task easier to perform" [CT, Project manager]. The project also divided the whole set of pictures into three pools: sign-up pool, working pool, and finished pool. The signup pool had 20 clusters (of three related pictures). The ideas was that a user who just signedup for tagging would start with this small pool. The tagging work on the sign-up pool would be scrapped as it served just for gaining experience. After a user finished ten clusters from the sign-up pool, the website would direct the user to the working pool. This pool includes the remaining pictures that need to be tagged, and thus is the main working zone. When a cluster had been tagged more than three times, it was considered finished and moved to the finished pool. Repeated tagging (three times) would help increase task quality.

While the task decomposition was quite well organised in the CT case, results aggregation was not clearly specified by the project and was considered future work by the interviewee. This model component was missing in the CT project.

Moving to the next component, crowd management included gathering users' demographic information and task allocation. CT collected demographic information at sign-up. The project assessed the task performance based on the sign-up pool, by comparing the users' tags with known correct answers. This allows the project making decision whether a user should work on the sign-up pool for training, or the working pool for actually performing the tasks. Yet, crowd management in CT is quite preliminary since the project did not build complete user profiles, which could be done if the project had built a metric to assess user performance.

Since tagging was performed by voluntary users, there was no guarantee that the results would be of high quality. Quality control is recommended by the model as necessary for projects similar to the CT case. In fact, two run-time quality control mechanisms were implemented in the CT project. The first mechanism asked the users directly how confident they were about tagging in order to identify the reliability level. The second mechanism was based on expert evaluation after all tags were received from the crowd. The choice of these mechanisms led to a concern that quality control "heavily depended on one person's opinions" [CT, Project manager]. As previously noted, the project did not address the concern and left it to future work.

To attract the crowd, project manager considered both extrinsic and intrinsic incentive mechanisms. However, he finally decided not to use the former so as he believed the users would be intrinsically keen enough to contribute to the citizen science project. As a result, the project was mainly based on intrinsic incentives. Similar to other citizen science projects (Brabham 2012), CT attracted participants who wished to make an altruistic contribution to science; as stated in the website "every image you tag will help us to better understand the relationships between New Zealand's invasive mammals and native species" [CT, website].

Regarding technical configuration, the project built a dedicated website to publicize the open call. This website also functioned as a crowdsourcing platform, managing pools and clusters, distributing pictures and collecting tags. The decision to develop a dedicated website was that the project members wanted to have full control over the entire set of crowdsourcing activities.

\section{Logo Design Contest (LDC)}

In the LDC case, the decision to crowdsource was based on two main reasons. The first reason was the ability of the crowd to provide diverse and innovative solutions. "The university has decided to conduct the open contest to find ideas that are standard [i.e. meeting requirements] and creative" [LDC, Project manager]. This is consistent with other crowdsourcing cases where open calls seek unique and innovative ideas (Brabham 2010; Leimeister et al. 2009). A second influence on the crowdsource decision was to call for designers from both inside and outside 
the university. Interestingly, reduced costs (compared to hiring a design expert) was not considered as an important factor in the decision to crowdsource.

Task design considered the defining of requirements for the logo, terms and conditions to join the contest, submission deadline, and prizes. Within these elements, the requirements played a major role as they specified what the solution should look like (Zheng et al. 2011). However, it seems that task description in LDC did not fully elucidate what had to be done, which led to an extension of the contest due to several requests to clarify requirements.

The workflow design was an interesting activity in the CT project with two distinctive aspects. First, while our model suggests task decomposition, the LDC project did not decompose the task. This can be explained by the nature of the logo design task, which seems difficult to break down into smaller tasks. Crowdsourcing a whole task has been already adopted in several design contests. Examples include a bus stop shelter design (Brabham 2012) and T-shirt design (Howe 2006). Second, LDC published its workflow description in the open call. According to the open call, the project workflow consisted of four steps: 1) the crowd submits their solutions (round 1); 2) a preliminarily evaluation is conducted by the board; 3) based on the board evaluation, a short-list of submissions is chosen, given feedback, and then re-submissions are allowed (round 2); and 4) the final submissions are evaluated, voted, ranked, and awarded, which is referred to as result aggregation in the model. This workflow description provided transparency to the process by explaining exactly what would happen during the project.

Crowd management was not a focus in the LDC case. The project did not assign tasks to any specific members or even profiled the crowd. The LDC project only profiled the participants in the second round, when a subset of submissions was already chosen. This was considered a project limitation: "the management of crowd information was limited, which may be because we did not specify rules about providing information” [LDC, Project Coordinator].

To control quality, the LDC project relied on expert evaluation (Zhao and Zhu 2014). In particular, the Board that aggregated results was also the Board that assessed quality and provided feedback to the participants. Since the number of submissions was not large (68 in total), this revealed an effective mechanism. However, the project found a few cheating submissions that were likely copied from logos of other organisations. These cases were identified by external experts on the Board, who have long experience with logo design.

The project adopted extrinsic mechanisms to attract the participants, which consisted of monetary rewards and recognition by the organisation. Like other contests, the monetary rewards were only provided for the best solutions, which included two creative prizes and one top winner prize. The creative and top winner prizes were quite valuable, relative to the living standard in the area, equivalent to one and five month's salary of a typical office worker, respectively. Another extrinsic motivation for the participants was that the project announced the winners on the university website, which is a recognition motivator (Brabham 2012). Both types of motivations were clearly aligned with our model.

The technical configuration was rather poor in this project, as it only used the website as a channel to publish the open call of the contest. Email was used to receive the submissions. This was because the project members were not aware of existing technology supporting crowdsourcing contests.

Overall, the two cases suggested the adequacy of the proposed model to describe the project activities. Adequateness was indicated through the highly matching between the model components and the project activities. Adequateness was further suggested through the participant interviews. When we showed graphical representations of the project using the model, the participants viewed the model to be aligned with their own representations. As one participant noted, "we may miss some of the points, but we touch all of them [all model components]" [CT, Project manager]. 


\subsubsection{Perceived Utility of the Model}

We analyse the utility of the model based on the interviewees' perception of how useful it seems to help planning and running the project. This is mainly based on' judgements and comments of project leaders and coordinators. In general, the result from the analysis suggest the model to be a useful tool for crowdsourcing. This was demonstrated by the following comments.

"I think it will be nice to follow the model. [...]. Yes, I want to use the model, following this flow or at least have something to follow" [CT, Project manager]

"The model is very well constructed and all of its activities should be necessarily for the project" [LDC, Coordinator]

"As I said, I think this model is totally suitable" [LDC, Project Manager]

Finding the usefulness of the model, these participants were extremely enthusiastic about applying the model for the future crowdsourcing projects:

"I think that any future crowdsourcing projects should apply strictly these steps, which will create better results" [LDC, Coordinator]

"From my opinion, the model can be suitable for many activities that need the resources from the crowd" [LDC, Project Manager]

Interestingly, we found slightly different views between the project manager and coordinator roles over what aspects of the model were most useful. For instance, in the LDC case, while the project manager viewed the model as a tool for making decisions, the project coordinator instead stressed the role of the model in supporting communication among project members and in achieving a consensus. These differences suggest the model's utility was not confined to one particular role.

In summary, we conducted two case studies evaluating the process model. The results of the case studies found evidence that the model can represent the key activities of crowdsourcing projects. Within each project, the model components were demonstrated and instantiated in the project contexts, although small differences in the instantiation could be found. Furthermore, we also obtained evidence of the perceived usefulness of the model, inspired by the reception of the key project informants.

\section{Discussion}

Multiple efforts conceptualising the crowdsourcing process have been reported in the literature. Most of them, however, view crowdsourcing processes as one-off activities, which are difficult to establish and reproduce (Amrollahi 2015). The issue of establishing crowdsourcing as a repeatable process becomes more pressing, since the crowdsourcing strategy has recently been integrated with some core organisational processes. This paper introduces a BPC lens on conceptualising crowdsourcing processes. While the term BPC has been used previously (La Vecchia and Cisternino 2010), it is this paper that elevates and defines the BPC concept. We suggest that BPC is best conceptualised as balancing between the business process construct and the crowdsourcing construct. Regarding the business process construct, BPC serves as a template for creating multiple, real life instances of the same crowdsourcing process.

The BPC conceptualisation can only stand with the condition that there are common repeatable activities of existing crowdsourcing processes. In this research, the condition has been met. Our scoping review found a set of common activities in crowdsourcing processes, repeatedly pointed out by multiple knowledge sources (Table 1). These common activities, which have been supported by other recent reviews (Amrollahi 2015; Hosseini et al. 2015), enable us to confirm the condition backing the BPC concept. Further, these common activities also suggest the basic building blocks of BPC. 
Using the building blocks identified by the scoping review, we then conceptualised BPC through a process model. The model, on the one hand, sharpens the BPC concept, using a process viewpoint that is well known to most organisations. On the other hand, the model highlights the core repeatable building blocks of BPC. With this focus, the model defines the abstract structure of BPC while treating new crowdsourcing projects as real life instances of the same core building blocks (Figure 1). All in all, the proposed process model brings the BPC concept into a space that is quite distinct from the one-off crowdsourcing endeavours (Lüttgens et al. 2014; Stol and Fitzgerald 2014).

The evaluation of the BPC process model in two existing crowdsourcing projects allowed us to test the representation and utility of the proposed model in practical environments. The case study analysis suggested high adequateness and usefulness of the BPC model in identifying the main activities of crowdsourcing processes. It can be observed from the results that the BPC model lays an abstract foundation of how organisations might rationally analyse and manage crowdsourcing projects. Furthermore, the case study results, to some extent, give insights into crowdsourcing project activities, which demonstrates how the model can be applied in practice.

We note however some limitations in the current study. Regarding the build activity, the model was constructed based on data gathered from a scoping review, and thus eventual limitations of these sources may also extend to this study (Kitchenham 2007). We partially addressed this concern by applying the 'wisdom of researchers', which is further discussed here to clarify its role in the current study. Founding on the 'wisdom of the crowd', the 'wisdom of researchers' holds the following four conditions: diversity, independence, decentralisation, and aggregation, which collectively allows the crowd to be wiser than an individual expert (Surowiecki 2004). In particular, we ensured diversity of knowledge by searching sources from eight online bibliographic databases. Our review was also based on independent sources of knowledge. We did not restrict the analysis to any particular theory or viewpoint; instead, we allowed the model to emerge from the extant literature. Decentralisation is a distinctive characteristic of the crowdsourcing field, which is noted for mixing different research fields (Geiger and Schader 2014). Surowiecki (2004) defined aggregation as a mechanism turning individual opinions into a final outcome. Such view supports the empirical decision to only select model components that received support from at least 10 sources. Because all four conditions hold, the application of the 'wisdom of researchers' enabled us to build a model faithfully representing BPC.

Regarding the evaluation by case study, the selection of two case studies could also be considered as a limitation, since generalisation to a broader context may be difficult. That applies to every case study. We note however that the generalisation for case studies is obviously not statistical but analytical one, where our model was used as an analytical framework for investigating the empirical results of crowdsourcing projects (Rowley 2002). Because of the characteristics inherent to the case study approach, the model by no means provides a generalised account of crowdsourcing projects. Rather, it is abstract. This could be seen via the small differences among the detailed activities mapped in the two investigated projects. We note that on the other hand the abstract characteristic of the model is also its strength, as it can be applied to and adapted in different crowdsourcing project settings.

\section{Conclusions and Future Work}

We began our study with the observation that more and more organisations are adopting crowdsourcing within their business. Thus, there is a strong need for a model to guide organisations to establish repeatable crowdsourcing processes (Djelassi and Decoopman 2013; Lüttgens et al. 2014). Addressing this gap, we developed a model allowing organisations to structure and manage the main building blocks necessary to establish the crowdsourcing strategy. We evaluated the model in two existing projects. Results, to a certain extent of generalisation, indicate the model is useful in identifying and articulating the main crowdsourcing activities. 
From an academic point of view, our study adopted a broad perspective on what activities need to be considered when planning, designing and instantiating crowdsourcing processes, thus overcoming the "excessive ad-hoc-ness" criticism found in the crowdsourcing literature (Geiger and Schader 2014; Man-Ching et al. 2011). Although a few models and frameworks have already been proposed (Amrollahi 2015; Hetmank 2013), the current study is the first to both conceptualise and evaluate a BPC model. From a practical point of view, the proposed model suggests several components that need to be articulated when establishing crowdsourcing processes. This provides a blueprint for managers and process designers, guiding them in their crowdsourcing projects.

From a research point of view, our work extends the existing research in three aspects. First, the model construction was based on a comprehensive review, in terms of number of reviewed sources. We analysed 238 papers, compared to 39 papers in a model construction by Amrollahi (2015). Second, we allowed the model components to freely emerge from the literature, which introduced some new important components: 'the decision to crowdsource', 'workflow design', and 'task assignment'. Finally, the model was empirically evaluated using a case study approach. While our case study findings were mainly indicative, they moved forward the theoretical efforts conceptualising the crowdsourcing process (Amrollahi 2015; Hetmank 2013; Thuan et al. 2014).

Future work is needed to confirm the utility of the model across different crowdsourcing contexts. This confirmation can be done using a mix of evaluation techniques. We have also started developing a decision tool which will operationalise the model and provide further support to project managers and process designers. This development will contribute to a better understanding of the model interdependencies and their impact in crowdsourcing projects (Miah and Gammack 2014). Another interesting future research direction is to consider the model in the context of crowdsourcing instantiation, and in particular integration with existing crowdsourcing platforms. Although the model captures the main activities of crowdsourcing processes, it would be interesting to further study how the model could be used to automate process instantiations in specific crowdsourcing platforms. Giachetti (2004), for example, suggests four levels of focus related to such integration, which comprehend business processes, applications, data, and networks. Thus, future research should provide further detail about the model regarding information structures, data structures, and technical structures.

\section{References}

Afuah, A., and Tucci, C.L. 2012. "Crowdsourcing as a Solution to Distant Search" Academy of Management Review,37 (3). 355-375.

Allahbakhsh, M., Benatallah, B., Ignjatovic, A., Motahari-Nezhad, H.R., Bertino, E., and Dustdar, S. 2013. "Quality Control in Crowdsourcing Systems: Issues and Directions" Internet Computing, IEEE,17 (2). 76-81.

Allahbakhsh, M., Ignjatovic, A., Benatallah, B., Beheshti, S., Bertino, E., and Foo, N. 2012. "Reputation Management in Crowdsourcing Systems," 8th International Conference on Collaborative Computing: Networking, Applications and Worksharing (CollaborateCom), pp. 664-671.

Amrollahi, A. 2015. "A Process Model for Crowdsourcing: Insights from the Literature on Implementation" Proceedings of the Australasian Conference on Information Systems 2015,Paper 18.

Bonabeau, E. 2009. "Decisions 2.0: The Power of Collective Intelligence" MIT Sloan Management Review, 50 (2). 45-52.

Brabham, D.C. 2010. "Moving the Crowd at Threadless" Information, Communication \& Society,13 (8). 1122-1145. 
Brabham, D.C. 2012. "Motivations for Participation in a Crowdsourcing Application to Improve Public Engagement in Transit Planning" Journal of Applied Communication Research,40 (3). 307-328.

Brabham, D.C. 2013. Crowdsourcing. Canbridge, MA: The MIT Press.

Chandler, D., and Kapelner, A. 2013. "Breaking Monotony with Meaning: Motivation in Crowdsourcing Markets" Journal of Economic Behavior \& Organization, 9o. 123-133.

Cooper, R.G. 2008. "Perspective: The Stage-Gate® Idea-to-Launch Process-Update, What's New, and Nexgen Systems*" Journal of Product Innovation Management, 25 (3). 213232.

Dibbern, J., Goles, T., Hirschheim, R., and Jayatilaka, B. 2004. "Information Systems Outsourcing: A Survey and Analysis of the Literature" ACM SIGMIS Database,35 (4). 6102.

Djelassi, S., and Decoopman, I. 2013. "Customers' Participation in Product Development through Crowdsourcing: Issues and Implications" Industrial Marketing Management,42 (5). 683-692.

Doan, A., Ramakrishnan, R., and Halevy, A.Y. 2011. "Crowdsourcing Systems on the WorldWide Web" Communications of the ACM,54 (4). 86-96.

Eickhoff, C., and De Vries, A. 2013. "Increasing Cheat Robustness of Crowdsourcing Tasks" Information Retrieval,16 (2). 121-137.

Geiger, D., and Schader, M. 2014. "Personalized Task Recommendation in Crowdsourcing Information Systems-Current State of the Art" Decision Support Systems, 65. 3-16.

Geiger, D., Seedorf, S., Schulze, T., Nickerson, R.C., and Schader, M. 2011. "Managing the Crowd: Towards a Taxonomy of Crowdsourcing Processes" Proceedings of the Seventeenth Americas Conference on Information Systems. Paper 430.

Giachetti, R.E. 2004. "A Framework to Review the Information Integration of the Enterprise" International Journal of Production Research,42 (6). 1147-1166.

Gill, A.Q., Alam, S.L., and Eustace, J. 2015. "Social Architecture: An Emergency Management Case Study" Australasian Journal of Information Systems,19. 23-40.

Hetmank, L. 2013. "Components and Functions of Crowdsourcing Systems-a Systematic Literature Review," in: 11th International Conference on Wirtschaftsinformatik. Leipzig, Germany.

Hevner, A., and Chatterjee, S. 2010. Design Research in Information Systems: Theory and Practice. Integrated Series in Information Systems. Springer Berlin Heidelberg.

Hevner, A., March, S.T., Park, J., and Ram, S. 2004. "Design Science in Information Systems Research" MIS Quarterly,28 (1). 75-105.

Hirth, M., Hoßfeld, T., and Tran-Gia, P. 2012. "Analyzing Costs and Accuracy of Validation Mechanisms for Crowdsourcing Platforms" Mathematical and Computer Modelling,57 (11-12). 2918-2932.

Hosseini, M., Phalp, K., Taylor, J., and Ali, R. 2015. "On the Configuration of Crowdsourcing Projects" International Journal of Information System Modeling and Design (IJISMD), 6 (3). 27-45.

Howe, J. 2006. "The Rise of Crowdsourcing," in: Wired Magazine 20o6. Dorsey Press, pp. 14.

Ipeirotis, P.G., Provost, F., and Wang, J. 2010. "Quality Management on Amazon Mechanical Turk" Proceedings of the ACM SIGKDD workshop on human computation. 64-67. 
Jonker, J., and Pennink, B.W. 2010. "Conceptual Models," in: The Essence of Research Methodology. Springer Berlin Heidelberg, pp. 43-63.

Kaufmann, N., Schulze, T., and Veit, D. 2011. "More Than Fun and Money. Worker Motivation in Crowdsourcing-a Study on Mechanical Turk" Proceedings of the Seventeenth Americas Conference on Information Systems, Detroit, MI. Paper 340.

Khazankin, R., Satzger, B., and Dustdar, S. 2012a. "Optimized Execution of Business Processes on Crowdsourcing Platforms" IEEE 8th International Conference on Collaborative Computing: Networking, Applications and Worksharing. $443-451$.

Khazankin, R., Satzger, B., and Dustdar, S. 2012b. "Predicting Qos in Scheduled Crowdsourcing," in: Advanced Information Systems Engineering, J. Ralyté, X. Franch, S. Brinkkemper and S. Wrycza (eds.). Springer Berlin Heidelberg, pp. 460-472.

King, N. 2012. "Doing Template Analysis" Qualitative organizational research: Core methods and current challenges. 426-450.

Kitchenham, B. 2007. "Guidelines for Performing Systematic Literature Reviews in Software Engineering" Ver. 2.3 EBSE Technical Report.

Kittur, A., Nickerson, J., Bernstein, M., Gerber, E., Shaw, A., Zimmerman, J., Lease, M., and Horton, J. 2013. "The Future of Crowd Work" Proceedings of the 2013 Conference on Computer Supported Cooperative Work.

Kittur, A., Smus, B., Khamkar, S., and Kraut, R.E. 2011. "Crowdforge: Crowdsourcing Complex Work" Proceedings of the 24th annual ACM symposium on User interface software and technology. 43-52.

Kulkarni, A., Can, M., and Hartmann, B. 2012. "Collaboratively Crowdsourcing Workflows with Turkomatic" Proceedings of the ACM 2012 Conference on Computer Supported Cooperative Work. 1003-1012.

La Vecchia, G., and Cisternino, A. 2010. "Collaborative Workforce, Business Process Crowdsourcing as an Alternative of Bpo," in: Icwe201O. Lncs, F. Daniel and F.M. Facca (eds.). Springer, pp. 425-430.

Leimeister, J.M., Huber, M., Bretschneider, U., and Krcmar, H. 2009. "Leveraging Crowdsourcing: Activation-Supporting Components for It-Based Ideas Competition" Journal of Management Information Systems, 26 (1). 197-224.

Little, G., Chilton, L.B., Goldman, M., and Miller, R.C. 2010. "Turkit: Human Computation Algorithms on Mechanical Turk" Proceedings of the 23nd annual ACM symposium on User interface software and technology. 57-66.

Lüttgens, D., Pollok, P., Antons, D., and Piller, F. 2014. "Wisdom of the Crowd and Capabilities of a Few: Internal Success Factors of Crowdsourcing for Innovation" Journal of Business Economics, 84 (3). 339-374.

Malone, T.W., Laubacher, R., and Dellarocas, C. 2010. "The Collective Intelligence Genome" IEEE Engineering Management Review,38 (3). 38- 52.

Man-Ching, Y., King, I., and Kwong-Sak, L. 2011. "A Survey of Crowdsourcing Systems" 2011 IEEE third international conference on Privacy, security, risk and trust (passat), and 2011 IEEE third international conference on social computing (socialcom). 766-773.

Mason, W., and Watts, D.J. 2009. "Financial Incentives and the "Performance of Crowds"'" Proceedings of the ACM SIGKDD Workshop on Human Computation. 77-85.

Miah, S.J., and Gammack, J.G. 2014. "Ensemble Artifact Design for Context Sensitive Decision Support" Australasian Journal of Information Systems, 18 (2).

Miles, M.B., Huberman, A.M., and Saldaña, J. 2014. Qualitative Data Analysis: A Methods Sourcebook. SAGE Publications, Incorporated. 
Muhdi, L., Daiber, M., Friesike, S., and Boutellier, R. 2011. "The Crowdsourcing Process: An Intermediary Mediated Idea Generation Approach in the Early Phase of Innovation" International Journal of Entrepreneurship and Innovation Management,14 (4). 315332.

Muntés-Mulero, V., Paladini, P., Manzoor, J., Gritti, A., Larriba-Pey, J.L., and Mijnhardt, F. 2013. "Crowdsourcing for Industrial Problems," in: Citizen in Sensor Networks. Lncs, J. Nin and D. Villatoro (eds.). Springer Berlin Heidelberg, pp. 6-18.

Paré, G., Trudel, M.-C., Jaana, M., and Kitsiou, S. 2015. "Synthesizing Information Systems Knowledge: A Typology of Literature Reviews" Information \& Management,52. 183199.

Park, S., Shoemark, P., and Morency, L.-P. 2014. "Toward Crowdsourcing Micro-Level Behavior Annotations: The Challenges of Interface, Training, and Generalization" Proceedings of the 19th international conference on Intelligent User Interfaces. 37-46.

Pedersen, J., Kocsis, D., Tripathi, A., Tarrell, A., Weerakoon, A., Tahmasbi, N., Jie, X., Wei, D., Onook, O., and De Vreede, G.J. 2013. "Conceptual Foundations of Crowdsourcing: A Review of Is Research" 46th Hawaii International Conference on System Sciences (HICSS). 579-588.

Rosen, P.A. 2011. "Crowdsourcing Lessons for Organizations" Journal of Decision Systems,20 (3). 309-324.

Rouse, A.C. 2010. "A Preliminary Taxonomy of Crowdsourcing" Proceedings of the 21st Australasian Conference on Information Systems. Paper 76.

Rowley, J. 2002. "Using Case Studies in Research" Management research news,25 (1). 16-27.

Satzger, B., Psaier, H., Schall, D., and Dustdar, S. 2011. "Stimulating Skill Evolution in MarketBased Crowdsourcing," in: Bpm 2011. Lncs, S. Rinderle-Ma, F. Toumani and K. Wolf (eds.). Springer Berlin Heidelberg, pp. 66-82.

Saxton, G.D., Oh, O., and Kishore, R. 2013. "Rules of Crowdsourcing: Models, Issues, and Systems of Control" Information Systems Management,30 (1). 2-20.

Schenk, E., and Guittard, C. 2011. "Towards a Characterization of Crowdsourcing Practices" Journal of Innovation Economics,7 (1). 93-107.

Stewart, O., Lubensky, D., and Huerta, J.M. 2010. "Crowdsourcing Participation Inequality: A Scout Model for the Enterprise Domain" Proceedings of the ACM SIGKDD Workshop on Human Computation. 30-33.

Stol, K.-J., and Fitzgerald, B. 2014. "Two's Company, Three's a Crowd: A Case Study of Crowdsourcing Software Development" Proceedings of the 36th International Conference on Software Engineering. 187-198.

Surowiecki, J. 2004. The Wisdom of Crowds: Why the Many Are Smarter Than the Few and How Collective Wisdom Shapes Business. New York: Doubleday.

Thuan, N.H., Antunes, P., and Johnstone, D. 2014. "Toward a Nexus Model Supporting the Establishment of Business Process Crowdsourcing," in: Fdse 2014. Lncs, T.K. Dang, R. Wagner, E. Neuhold, M. Takizawa, J. Küng and N. Thoai (eds.). Springer, Heidelberg, pp. 136-150.

Thuan, N.H., Antunes, P., and Johnstone, D. 2015. "A Design Science Method for Emerging Decision Support Environments" Proceedings of the Australasian Conference on Information Systems 2015,Paper 32.

Thuan, N.H., Antunes, P., and Johnstone, D. 2016. "Factors Influencing the Decision to Crowdsource: A Systematic Literature Review" Information Systems Frontiers,18 (1). 47-68. 
Tokarchuk, O., Cuel, R., and Zamarian, M. 2012. "Analyzing Crowd Labor and Designing Incentives for Humans in the Loop" IEEE Internet Computing Magazine,16 (5). 45-51.

Tranquillini, S., Daniel, F., Kucherbaev, P., and Casati, F. 2015. "Modeling, Enacting, and Integrating Custom Crowdsourcing Processes" ACM Transactions on the Web (TWEB), 9 (2). Article 7.

van der Aalst, W., and Hee, K.M. 2004. Workflow Management: Models, Methods, and Systems. Cambridge, MA: The MIT Press.

Venable, J., Pries-Heje, J., and Baskerville, R. 2012. "A Comprehensive Framework for Evaluation in Design Science Research" Design Science Research in Information Systems. Advances in Theory and Practice. 423-438.

Vukovic, M., and Bartolini, C. 2010. "Towards a Research Agenda for Enterprise Crowdsourcing," in: Leveraging Applications of Formal Methods, Verification, and Validation. Lncs, T. Margaria and B. Steffen (eds.). Springer Berlin Heidelberg, pp. 425434 .

Vukovic, M., Laredo, J., and Rajagopal, S. 2010. "Challenges and Experiences in Deploying Enterprise Crowdsourcing Service," in: Icwe 2010. Lncs, B. Benatallah, F. Casati, G. Kappel and G. Rossi (eds.). Springer Berlin Heidelberg, pp. 460-467.

Webster, J., and Watson, R.T. 2002. "Analyzing the Past to Prepare for the Future: Writing a Literature Review" MIS Quarterly,26 (2). xiii-xxiii.

Wexler, M.N. 2011. "Reconfiguring the Sociology of the Crowd: Exploring Crowdsourcing" International Journal of Sociology and Social Policy,31 (1/2). 6-20.

Yin, R.K. 2013a. Case Study Research: Design and Methods. Sage publications.

Yin, R.K. 2013b. "Validity and Generalization in Future Case Study Evaluations" Evaluation,19 (3). 321-332.

Zhao, Y., and Zhu, Q. 2014. "Evaluation on Crowdsourcing Research: Current Status and Future Direction" Information Systems Frontiers,16 (3). 417-434.

Zheng, H., Li, D., and Hou, W. 2011. "Task Design, Motivation, and Participation in Crowdsourcing Contests" International Journal of Electronic Commerce, 15 (4). 57-88.

Copyright: (C) 2017 Thuan, Antunes \& Johnstone. This is an open-access article distributed under the terms of the Creative Commons Attribution-NonCommercial 3.0 Australia License, which permits non-commercial use, distribution, and reproduction in any medium, provided the original author and AJIS are credited.

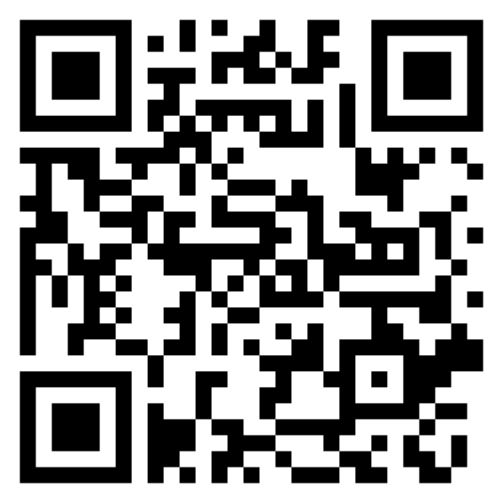

